\title{
Altered Expression of RB and pRB in Tissue Arrays of Primary Breast Cancers and Matched Axillary Lymph Node Metastases
}

\author{
Carmen Leser $\mathbb{D}^{\mathrm{D}},{ }^{1}$ Angelika Reiner, ${ }^{2}$ Georg Dorffner, ${ }^{3}$ Marie-Theres Kastner, ${ }^{1}$ Martin Igaz, ${ }^{4}$ \\ Christian Singer, ${ }^{1}$ Deirdre Maria König-Castillo, ${ }^{5}$ Christine Deutschmann, ${ }^{1}$ Daniel König, ${ }^{6}$ \\ Iris Holzer, ${ }^{1}$ and Daphne Gschwantler-Kaulich ${ }^{1}$ \\ ${ }^{1}$ Department of Obstetrics and Gynecology, Cancer Comprehensive Center, Medical University of Vienna, Vienna, Austria \\ ${ }^{2}$ Department of Pathology, Danube Hospital, Vienna, Austria \\ ${ }^{3}$ Section for Artificial Intelligence and Decision Support, Medical University of Vienna, Vienna, Austria \\ ${ }^{4}$ Laboratory Dr. Kosak, Vienna, Austria \\ ${ }^{5}$ Department of Urology, Medical University of Vienna, Vienna, Austria \\ ${ }^{6}$ Clinical Division of Social Psychiatry, Department of Psychiatry and Psychotherapy, Medical University of Vienna, \\ Vienna, Austria
}

Correspondence should be addressed to Carmen Leser; carmen.leser@meduniwien.ac.at

Received 28 November 2021; Revised 9 February 2022; Accepted 11 February 2022; Published 4 March 2022

Academic Editor: Neslihan Cabio Lu

Copyright ( $\odot 2022$ Carmen Leser et al. This is an open access article distributed under the Creative Commons Attribution License, which permits unrestricted use, distribution, and reproduction in any medium, provided the original work is properly cited.

Objectives. The retinoblastoma (RB) pathway is crucial in the development and progression of many cancers. To better understand the biology of progressive breast cancer $(\mathrm{BC})$, we examined protein expression of the RB pathway in primary BCs and matched axillary lymph node metastases (LM). Methods. Immunohistochemistry was used to evaluate cyclin D1, CDK4/6, RB, phosphorylated RB (pRB), and E2F1 expression in tissue arrays containing cores of 50 primary BCs and matched LM. The number of positive tumor cells and staining intensity were scored. Results. The proteins were localized in the nucleus, while CDK6 was detected in the cytoplasm and CDK4 was found in both. pRB and E2F1 showed higher expression in matched LM than in primary tumors. Expression of these proteins differed significantly by the percentage of positive tumor cells, while proteins in the proximal portion of the RB pathway showed no significant differences. The main path of alteration consisted of high pRB in primary BC, remaining $\mathrm{pRB}$ high in the majority of LM, variations occurring in fewer cases. All matched LM of the few primary tumors that had unaltered RB and pRB expression showed changes in RB or pRB expression. Conclusion. Expression of pRB and E2F1 was significantly higher in LM than in primary BC. A majority of cancers with LM showed altered RB or pRB expression, suggesting that proteins downstream in the $\mathrm{RB}$ pathway play a critical role in metastatic $\mathrm{BC}$ and disease progression. So looking at the $\mathrm{RB}$ pathway could be an option for chemotherapy decisions in patients with only few LM.

\section{Introduction}

Accounting for $25 \%$ of malignant neoplasms in women worldwide, breast cancer (BC) is the most common malignant tumor in women [1]. The retinoblastoma (RB) pathway (Figure 1) plays a critical role in all types of BCs; however, the mechanisms by which the pathway is inactivated vary. Luminal $\mathrm{BC}$ is the most common subtype of $\mathrm{BC}$ and is treated with endocrine therapy or surgical resection. In most cases, luminal $\mathrm{BC}$ has a favorable prognosis with no recurrence, but approximately $30 \%$ of these patients suffer from it. It may be due to the development of endocrine resistance and evasion of endogenous growth suppressors [2]. The mechanism underlying such a recurrence is not fully understood, but alterations in tumor pathways and cell cycle checkpoints are known to contribute [3]. Dysregulation of CDK4/6 is a common phenomenon in luminal BC recurrence and is a result of alterations in the $\mathrm{RB}$ pathway caused by increased expression of cyclin D1 [4]. Based on this, therapeutics targeting the cyclin D-CDK4/6-Rb pathway 
have gained interest as treatments for luminal BC [5]. Her2 positive $\mathrm{BC}$ is often associated with cyclin D1 deregulation, but rarely with RB loss [6]. Contrarily, dysregulation of the $\mathrm{RB}$ pathway is caused mainly through $\mathrm{RB}$ loss in triplenegative $\mathrm{BC}$ (TNBC), and $\mathrm{RB}$ loss in TNBC is associated with good responses to chemotherapy [7].

Despite these interesting findings, neither clinical use nor standardized methodologies for analysis of RB pathway deregulation have been established. Surprisingly, there have been few analyses of the RB pathway in clinical specimens. While some data showing RB pathway deregulation in early $\mathrm{BC}$ such as in ductal carcinoma in situ, does exist [8], RB pathway disruption in metastasized $\mathrm{BC}$ has not been described to the best of our knowledge. Therefore, we investigated the expression of proteins in the proximal and downstream portions of the RB pathway in lymphnode positive tumors, comparing primary $\mathrm{BC}$ and $\mathrm{LM}$.

\section{Methods}

2.1. Patient and Tumor Characteristics. A total of 47 invasive ductal carcinomas and three invasive lobular carcinomas were examined. The clinicopathological parameters of the tumors were provided by the distributor and are detailed in Table 1. Altered expression of RB and pRB was assumed as defined by Gravessea et al. [9].

2.2. Immunohistochemistry. All markers were investigated by immunohistochemistry (IHC) on formalin fixed paraffin embedded slides containing 50 tissue invasive BCs and 50 matched LM cores using commercially available tissue microarrays (BR 10010e, US Biomax Inc., Rockville, MD, USA). Tissue microarrays consisted of duplicate cores per case with $1 \mathrm{~mm}$ in diameter each.

Tumor characterization for steroid hormone receptors and immunohistochemical scoring for HER-2 were used as given by the provider. Data for gene amplification was not given. Thus, cases with equivocal HER-2 scoring were excluded for some correlations in results.

Discrepancies of case numbers are due to missing values because some tissue cores came off the slides during laboratory processing.

All IHC analyses were performed on a Ventana ${ }^{\circledR}$ BenchMark System using the OptiView DAB IHC Detection Kit (Ventana Medical Systems, Inc., Oro Valley, Arizona, USA). Incubation omitting primary antibodies was used as a negative control. Descriptions of primary antibodies are provided in Table 2. Antigen retrieval before application of the first antibody was carried out by heat incubation $\left(37^{\circ} \mathrm{C}\right)$. As indicated in Table 2, some of the antibodies required additional incubation per the instructions of the Ventana OptiView Amplification Kit.

Microscopic analysis was performed by scoring the number of positive tumor cells semiquantitatively for all markers. The percentage of positive tumor cells was normalized to the total number of tumor cells. Stromal cells like fibroblasts or nontumorigenic tissue were not taken into account. Samples were divided into three groups based on



FIGURE 1: Cyclin D cyclin-dependent kinase $4 / 6$ retinoblastoma pathway.

TABle 1: Clinicopathologic parameters.

\begin{tabular}{|c|c|}
\hline Clinicopathological characteristics & $n(\%)$ \\
\hline Median age in years (IQR) & $50(44-55)$ \\
\hline \multicolumn{2}{|l|}{ Histological subtype } \\
\hline Invasive ductal carcinoma & $47(94 \%)$ \\
\hline Invasive lobular carcinoma & $3(6 \%)$ \\
\hline \multicolumn{2}{|l|}{ Grade } \\
\hline $\mathrm{X}$ & $6(12 \%)$ \\
\hline 1 & $7(14 \%)$ \\
\hline 2 & $28(54 \%)$ \\
\hline 3 & $9(18 \%)$ \\
\hline \multicolumn{2}{|l|}{ Tumor stage } \\
\hline $\mathrm{T} 1$ & $1(2 \%)$ \\
\hline $\mathrm{T} 2$ & $32(64 \%)$ \\
\hline $\mathrm{T} 3$ & $10(20 \%)$ \\
\hline $\mathrm{T} 4$ & $7(14 \%)$ \\
\hline \multicolumn{2}{|l|}{ Nodal status } \\
\hline N1 & $29(58 \%)$ \\
\hline $\mathrm{N} 2$ & $20(40 \%)$ \\
\hline N3 & $1(2 \%)$ \\
\hline \multicolumn{2}{|l|}{ Receptors } \\
\hline Triple negative & $16(32 \%)$ \\
\hline Hormone receptor positive (mainly ER, partly PR) & $19(38 \%)$ \\
\hline HER-2 equivocal (score $2+$ ) & $9(18 \%)$ \\
\hline HER-2 positive (score $3+$ ) & $5(10 \%)$ \\
\hline Unknown & $1(2 \%)$ \\
\hline
\end{tabular}

the number of stained tumor cells: negative ( $0 \%$ of tumor cells stained), low ( $\leq 50 \%$ of tumor cells stained), and high ( $>50 \%$ of tumor cells stained). Nuclear staining was scored for cyclin D1, CDK4, RB, pRB, and E2F1. CDK6 showed only cytoplasmic staining. Any positive staining was considered a positive result. In a few cases with discrepancies of immunohistochemical staining, higher protein expression in either core was considered for the result.

2.3. Statistical Analysis. This study was strictly exploratory in nature; thus, no correction for multiple testing was applied. A significance level of $\alpha=0.05$ was used for reporting and interpretation of clinical plausibility and consistency of results.

Statistical analyses were performed to determine the statistical significance of observed differences in staining between the primary tumor and corresponding lymph node metastases for each antibody. Where significant differences were observed, intergroup differences in BC subgroups (i.e., TNBC, ER, PR, or HER-2 positive tumors) were investigated. 
TABle 2: Primary antibodies used in immunohistochemistry.

\begin{tabular}{lcccc}
\hline Antibody & Clone & Dilution & Company & Pretreatment \\
\hline Cyclin D1 & SP4-R & Ready to use & Ventana $^{\circledR}$ Medical Systems & CC1 Ventana ${ }^{\circledR}$ Medical Systems \\
CDK4 & DCS-156 & $1: 5$ & CZytomed Systems & CC1 and OptiView Amplification Kit \\
RB & 1F8 (Rb1) & $1: 50$ & Thermo Fisher Scientific & CC1 and OptiView Amplification Kit \\
pRB & pThr826 & $1: 100$ & Thermo Fisher Scientific & CC1 and OptiView Amplification Kit \\
E2F1 & KH95 & $1: 80$ & Thermo Fisher Scientific & CC1 and OptiView Amplification Kit \\
\hline
\end{tabular}
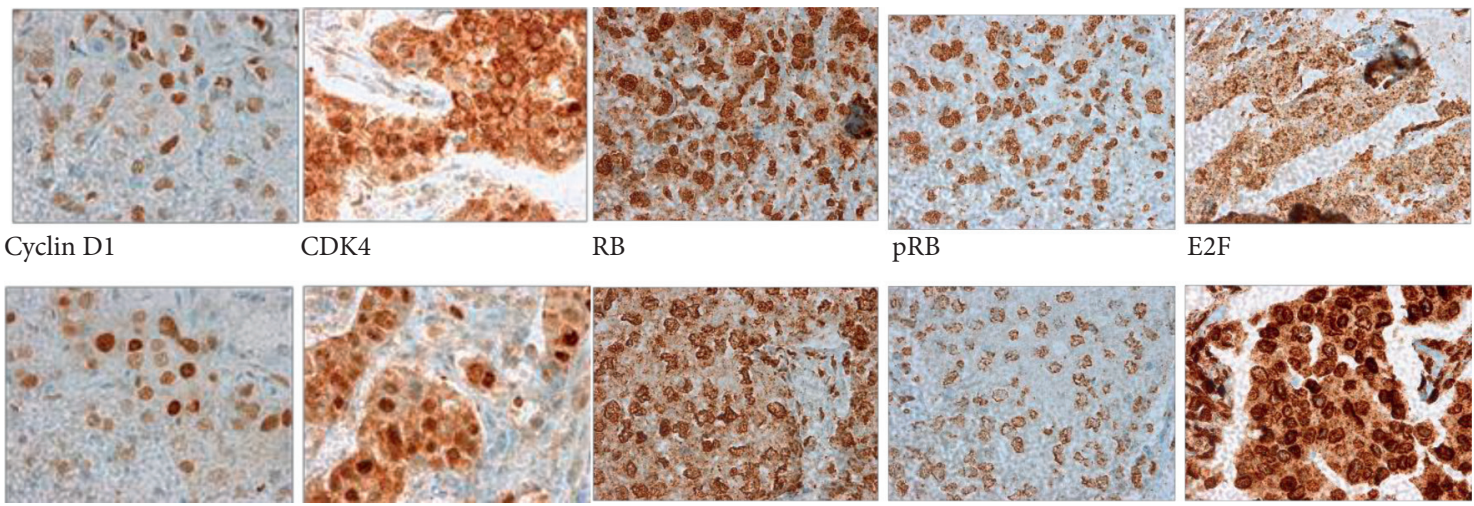

FIGURE 2: Immunohistochemical staining of primary breast cancer and matched axillary lymph node metastases. Original magnification $\mathrm{x} 400$. First row shows primary tumors, and second row shows matched axillary lymph node metastases.

Either the chi-squared test or Fisher's exact tests were used to assess intergroup differences in expression. The associations between the expression of tested markers and clinicopathological parameters were analyzed by Spearman's Rho test. All calculations were performed using IBM SPSS Statistics version 23 (IBM Corp., Armonk, NY).

\section{Results}

All proteins were present in the nucleus (Figure 2). Expression of cyclin D1 and CDK4 was significantly correlated (correlation coefficient 0.4).

Positive expression of proteins in the proximal $\mathrm{RB}$ pathway (cyclin D1, CDK4, and RB) was comparable. However, proteins in the downstream portion of the RB pathway (pRB, E2F1) showed significantly higher positivity in LM than in BC (Table 3). In the majority of primary BCs and LM, pRB was expressed at high levels, but above $50 \%$. The majority of cases in E2F1 showed expression at low levels, below $50 \%$. Altered expression of RB and pRB was observed in 43 primary $\mathrm{BC}$, reflecting a disrupted $\mathrm{RB}$ pathway. The alteration to the RB pathway was due to RB loss in 23 primary BCs (negative immunohistochemical staining) and high levels of pRB expression in 21 primary BCs (Table 4). Only one of the cancers with altered expression had both RB loss and high positivity for $\mathrm{pRB}$. The change of $R B$ and $p R B$ from primary tumor to lymph node metastasis is depicted in Figure 3. In primary BC with high levels of $\mathrm{pRB}$ expression, $\mathrm{pRB}$ expression was also high in matched axillary lymph node metastases. In contrast, primary $\mathrm{BC}$ with $\mathrm{RB}$ loss showed a variety of changes in LM at comparable frequencies. All primary $\mathrm{BC}$ with unaltered $\mathrm{RB}$ or $\mathrm{pRB}$ showed altered $\mathrm{RB}$ or $\mathrm{pRB}$ in all matched LM. In
TABLE 3: RB pathway proteins in primary BC compared with LM.

\begin{tabular}{lccccc}
\hline & \multicolumn{2}{c}{ Primary cancer } & \multicolumn{2}{c}{ LM } & \multirow{2}{*}{$\chi^{2}$} \\
& Positive & Negative & Positive & Negative & \\
\hline Cyclin D1 & $43(86 \%)$ & $7(14 \%)$ & $38(83 \%)$ & $8(17 \%)$ & Ns \\
CDK4 & $28(56 \%)$ & $22(44 \%)$ & $21(42 \%)$ & $29(58 \%)$ & Ns \\
RB & $27(54 \%)$ & $23(46 \%)$ & $32(64 \%)$ & $18(36 \%)$ & Ns \\
pRB & $27(54 \%)$ & $23(46 \%)$ & $43(86 \%)$ & $7(14 \%)$ & 0.0004 \\
E2F1 & $33(69 \%)$ & $15(31 \%)$ & $43(86 \%)$ & $7(14 \%)$ & 0.041 \\
\hline
\end{tabular}

TABLE 4: Retinoblastoma pathway proteins with significantly different expression in primary breast cancer and matched axillary lymph node metastases.

\begin{tabular}{ccccccc}
\hline \multicolumn{4}{c}{ Primary cancer } & \multicolumn{3}{c}{ LM } \\
& 0 & $<50 \%$ & $>50 \%$ & 0 & $<50 \%$ & $>50 \%$ \\
\hline \multirow{2}{*}{ pRB } & 23 & $7(14 \%)$ & 20 & 7 & $6(12 \%)$ & 37 \\
& $(46 \%)$ & $(40 \%)$ & $(14 \%)$ & $34 \%)$ \\
E2F1 & 15 & 26 & $7(15 \%)$ & 7 & 35 & $8(16 \%)$ \\
& $(31 \%)$ & $(54 \%)$ & & $(14 \%)$ & $(70 \%)$ & 8 \\
\hline
\end{tabular}

Note. Different numbers are due to missing cores after staining.

subgroup analyses, cancers of all molecular subtypes exhibited altered RB ( 15 of 19 luminal, 5 of 5 HER-2 positive, 13 of 16 TNBC, and all 9 Her-2 equivocal cases). Only eight of the cancers showed unaltered expression of $\mathrm{RB}$ or $\mathrm{pRB}$ in primary $\mathrm{BC}$, most likely suggesting an intact $\mathrm{RB}$ pathway. Among these, four were ER negative and four were ER positive. Among the matching LM, seven showed high expression of $\mathrm{pRB}$ and two showed RB loss. This suggests $\mathrm{RB}$ pathway disruption in lymph node metastases of primary BC cases with unaltered RB pathway protein expression.

Correlations between protein expression and tumor grade are provided in Table 5. Only proteins in the 


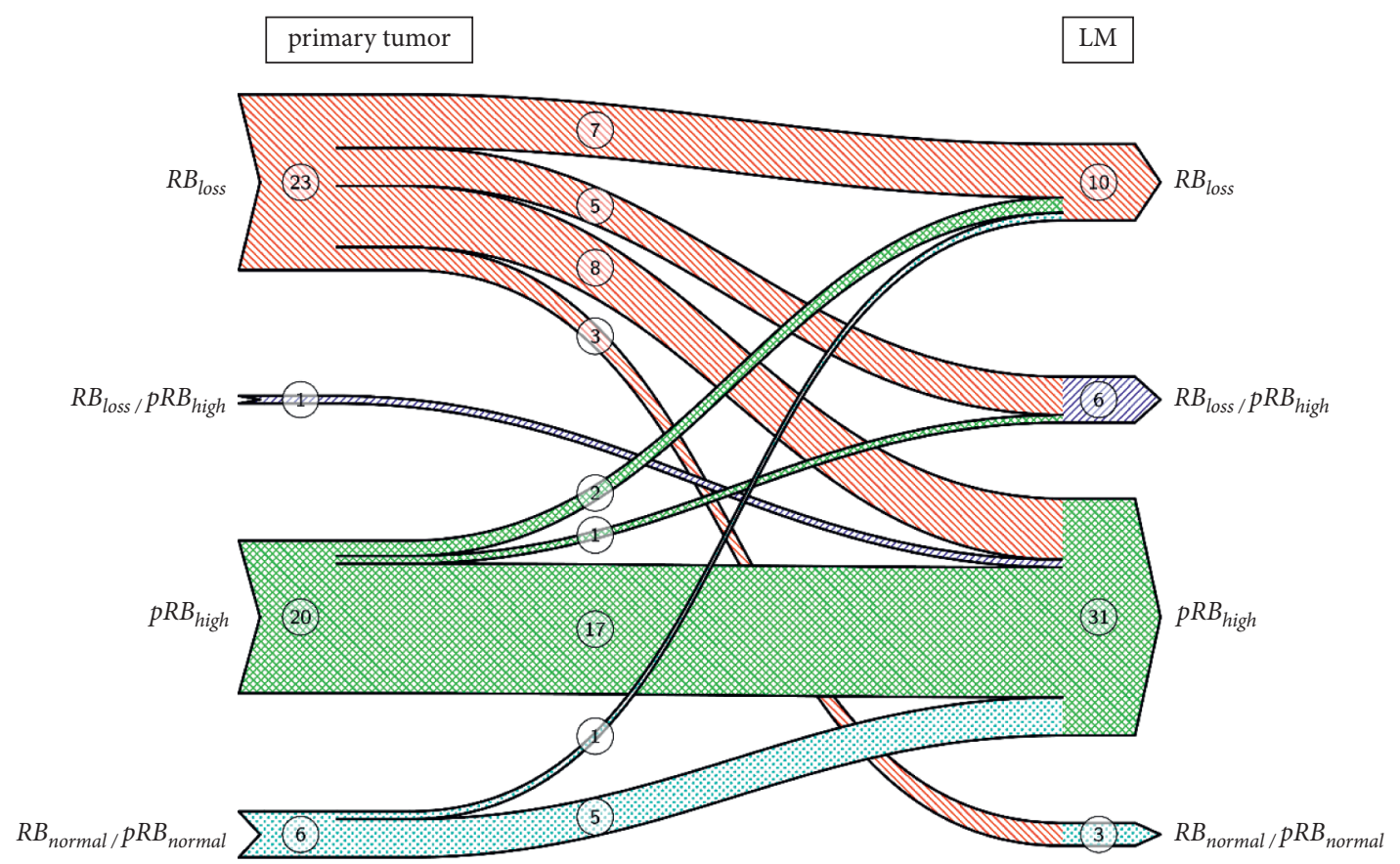

FIgURE 3: Change of RB and pRB from primary tumor to lymph node metastasis.

TABLE 5: RB pathway biomarkers in different grades of primary breast cancer tumors.

\begin{tabular}{|c|c|c|c|c|c|c|}
\hline \multirow{2}{*}{ Primary BC } & \multicolumn{2}{|c|}{ Grade 1} & \multicolumn{2}{|c|}{ Grade 2} & \multicolumn{2}{|c|}{ Grade 3} \\
\hline & Positive & Negative & Positive & Negative & Positive & Negative \\
\hline Cyclin D1 & 6 & 1 & 25 & 3 & 7 & 2 \\
\hline CDK4 & 3 & 4 & 16 & 12 & 5 & 4 \\
\hline $\mathrm{RB}$ & 1 & 6 & 16 & 12 & 6 & 3 \\
\hline $\mathrm{pRB}$ & 0 & 7 & 16 & 12 & 8 & 1 \\
\hline E2F1 & 4 & 3 & 19 & 8 & 7 & 2 \\
\hline
\end{tabular}

downstream portion of the RB pathway (pRB and E2F1) showed different expressions in different tumors. $\mathrm{RB}$ and pRB were not expressed in G1 cancers and were equally expressed in G2 in the majority of G3 cancers presenting positive expression (Table 5).

Proteins in the downstream portion of the RB pathway ( $\mathrm{RB}$ and $\mathrm{pRB}$ ) were also evaluated based on the level of lymph node metastases (1-3 LM; N1; 4-9 LM, N2). The vast majority showed altered RB with no differences between N1 and N2 (Table 6).

An overwhelming majority of luminal cancers showed positivity for all proteins; this was also true for HER-2 negative cancers. In TNBCs, all proteins showed roughly comparable distributions (Table 7). For HER-2 Score 3+ and thus, most likely, HER-2 amplified BC, the number of cases was too small for conclusive interpretation.

\section{Discussion}

The $\mathrm{RB}$ protein functions as a tumor suppressor, and deregulation of the $\mathrm{RB}$ pathway plays a critical role in tumor development and disease progression. In its nonphosphorylated active form, RB binds to the transcription factor E2F1 to repress progression from the G1 to the S
TABLE 6: Protein expression of the downstream RB pathway in axillary lymph node metastasis.

\begin{tabular}{lcc}
\hline & $\mathrm{N} 1$ & $\mathrm{~N} 2$ \\
& $n=28$ & $n=20$ \\
\hline RB loss & 15 & 9 \\
pRB high positivity & 12 & 10 \\
Overlap & 1 & 1 \\
\hline
\end{tabular}

phase. Thus, RB is an important gatekeeper in cell growth [10]. Cyclin D1 binds and regulates CDK4, which deactivates numerous targets including RB. E2F1 dissociation from the $\mathrm{RB} / \mathrm{E} 2 \mathrm{~F}$ complex leads to loss of the growth-inhibiting function of RB (Figure 2) [3].

Interestingly, $\mathrm{RB}$ pathway disruption is an important part of the progression of intraductal $\mathrm{BC}$ and is therefore relevant to the early stages of invasive $\mathrm{BC}$ [8]. This suggests that changes in the RB pathway may be associated with malignant transformation, even in the early stages of $\mathrm{BC}$ development, and also play a significant role in tumor progression in later stages of disease. Changes in the RB pathway may also serve as therapeutic indicators, but no predictive test using $\mathrm{RB}$ pathway alteration has been established. Development of a single specific molecule test 
TABLE 7: RB pathway biomarkers in BC subtypes.

\begin{tabular}{|c|c|c|c|c|c|c|c|c|}
\hline & \multicolumn{2}{|c|}{ ER } & \multicolumn{2}{|c|}{ TNBC } & \multicolumn{4}{|c|}{ HER-2 } \\
\hline & \multirow[b]{2}{*}{ Positive } & \multirow[b]{2}{*}{ Negative } & \multirow[b]{2}{*}{ Positive } & \multirow[b]{2}{*}{ Negative } & \multicolumn{2}{|c|}{$0 / 1+$} & \multicolumn{2}{|c|}{$3+$} \\
\hline & & & & & Positive & Negative & Positive & Negative \\
\hline Cyclin D1 & 18 & 1 & 12 & 4 & 26 & 4 & 5 & 0 \\
\hline CDK4 & 14 & 5 & 6 & 10 & 16 & 14 & 1 & 4 \\
\hline $\mathrm{RB}$ & 12 & 7 & 9 & 7 & 17 & 13 & 2 & 3 \\
\hline $\mathrm{pRB}$ & 12 & 7 & 9 & 7 & 17 & 13 & 2 & 3 \\
\hline $\mathrm{E} 2 \mathrm{~F} 1$ & 15 & 3 & 8 & 7 & 19 & 10 & 3 & 2 \\
\hline
\end{tabular}

Note. Different numbers are due to missing values.

may be difficult due to the variability in the effects of $\mathrm{RB}$ pathway alterations associated with different BC subtypes. Despite this, monitoring RB pathway deregulation could be clinically relevant for the development of individualized treatments in the future. To contribute to a deeper understanding of $\mathrm{RB}$ pathway disruption, we have analyzed the expression of proteins in the RB pathway in primary $\mathrm{BC}$ and matched axillary lymph node metastases.

Our results showed a significantly higher number of samples that showed expression of $\mathrm{pRB}$ and $\mathrm{E} 2 \mathrm{~F} 1$ in greater than $50 \%$ of the samples in LM compared with primary BC tumors. This altered protein expression occurs downstream in the pathway and reflects enhanced cell cycle progression. In contrast, expression of proteins in the proximal pathway was not significantly different in the primary tumors and metastases. We also examined protein expression patterns reflective of an altered or disrupted pathway as described by Gavressea et al. [9]. In our study, the overwhelming majority of primary cancers had altered protein expression leading to either RB loss or high levels of pRB. Few primary cancers showed no alterations in protein expression, but all BCs that had no alterations in $\mathrm{RB}$ pathway protein expression exhibited altered RB or pRB in their matched LM lymph node metastases. In 2017, Gavressa et al. found that the p53 mediated sensitivity of breast cancer cells to chemotherapeutic agents appears to be driven mostly by pRB, and that using agents that enhance RB phosphorylation might possibly increase the chemosensitivity of breast cancer cells. This may corroborate the wide acceptance of chemotherapy as an adjuvant therapy regimen, especially chemotherapy in BC with axillary lymph node metastases. Our findings suggest that chemotherapy should be an option for patients with few lymph node metastases. Derenzini et al. have previously shown that human $\mathrm{BC}$ cells exhibiting RB loss have higher sensitivity to 5-FU and methotrexate [11]. However, some studies have found that low expression of RB in ER+/HER2+ patients leads to low pCR rates after neoadjuvant chemotherapy with anti-HER2 drugs $[12,13]$. Furthermore, in ER+/ HER2 negative $\mathrm{BC}, \mathrm{RB}$ loss of function is a predictive biomarker of resistance to palbociclib [14].

No significant correlations were observed between protein expression and tumor grade, but the majority of G3 primary tumors exhibited altered expression of $\mathrm{pRB}$ and E2F1, indicating enhanced cell cycle progression. Interestingly, this finding does not correlate with other literature showing that RB expression correlates with histologic grades 1 and 2 [15]. This difference can likely be explained by the fact that this earlier study examined only TNBCs, which are the most poorly differentiated subtype of BCs, whereas the current study included all molecular subtypes and tumor grades. Unlike earlier studies $[16,17]$, we did not observe any significant correlations between molecular tumor subtypes. The lack of correlations in our study may be due to the small number of samples of each subtype included. In the commercially available TMA, HER-2 score $2+$ cases were overrepresented compared to unselected $\mathrm{BC}$ cases. No data exist for these cases following HER-2 in situ hybridization results, according to the clinical guidelines. It should also be noted that in cases included in the commercially available TMAs, only small tumor samples are examined, which may result in negative staining in cases of pronounced protein expression heterogeneity. These shortcomings in our study may have contributed to the discrepancies between our results and earlier studies, mainly consisted of HER-2 of score $2+$ with unknown in situ hybridization results.

The results of our study are limited because of the small sample size and partly limited patient characteristics given by the provider of tissue microarrays. However, due to our promising findings, we plan a future study on the tissue of patients from our institution. In former studies, some markers have been shown to be quite heterogeneous in breast carcinomas. Some discrepancies between primary tumors and metastases could be due to tumor heterogeneity [18].

In conclusion, increased expression of $\mathrm{E} 2 \mathrm{~F}$ and $\mathrm{pRB}$ in lymph node metastasis compared with primary $\mathrm{BC}$ indicates that the downstream part of the cyclin D-CDK4/6-RB pathway is more often activated in metastatic tissue. In addition, a majority of cancers with axillary lymph node metastasis showed alterations of RB and $\mathrm{pRB}$. This suggests proteins downstream of the RB pathway play a critical role in metastatic breast cancer and progressive disease. Therefore, evaluation of the RB pathway may be prognostically relevant.

\section{Data Availability}

All the necessary data are included within the article.

\section{Ethical Approval}

Ethical approval for this study was not required.

\section{Conflicts of Interest}

The authors declare that there are no conflicts of interest. 


\section{Authors' Contributions}

Carmen Leser was responsible for writing and design and also helped with analysis. Angelika Reiner contributed to the writing and main analysis. Georg Dorffner carried out the statistics. Marie-Theres Kastner contributed to the IHC. Martin Igaz was responsible for analysis and test tissues. Christian Singer was responsible for design and helped with organization. Deirdre Maria Castillo contributed to the writing and helped with organization. Christine Deutschmann was responsible for the writing and analysis. Daniel König contributed to the writing and helped with organization. Iris Holzer contributed to the writing and design. Daphne Gschwantler-Kaulich contributed to the design and writing.

\section{References}

[1] L. A. Torre, F. Islami, R. L. Siegel, E. M. Ward, and A. Jemal, "Global cancer in women: burden and trends," Cancer Epidemiology Biomarkers \& Prevention, vol. 26, no. 4, pp. 444457, 2017.

[2] D. Hanahan and R. A. Weinberg, "Hallmarks of cancer: the next generation," Cell, vol. 144, no. 5, pp. 646-674, 2011.

[3] M. Ameratunga, E. Kipps, A. F. C. Okines, and J. S. Lopez, "To cycle or fight-CDK4/6 inhibitors at the crossroads of anticancer immunity," Clinical Cancer Research, vol. 25, no. 1, pp. 21-28, 2018.

[4] R. L. Sutherland and E. A. Musgrove, "Cyclins and breast cancer," Journal of Mammary Gland Biology and Neoplasia, vol. 9, no. 1, pp. 95-104, 2004.

[5] J. Ju, A.-J. Zhu, and P. Yuan, "Progress in targeted therapy for breast cancer," Chronic Diseases and Translational Medicine, vol. 4, no. 3, pp. 164-175, 2018.

[6] E. Shin, W. H. Jung, and J. S. Koo, "Expression of p16 and pRB in invasive breast cancer," International Journal of Clinical and Experimental Pathology, vol. 8, pp. 8209-8217, 2015.

[7] A. K. Witkiewicz, A. Ertel, J. McFalls, M. E. Valsecchi, G. Schwartz, and E. S. Knudsen, "RB-pathway disruption is associated with improved response to neoadjuvant chemotherapy in breast cancer," Clinical Cancer Research, vol. 18, no. 18 , pp. 5110-5122, 2012.

[8] E. S. Kndusen, T. F. Pajak, M. Qeenan et al., "Retinoblastoma and phosphate and tensin homolog tumor suppressors: impact on ductal carcinoma in situ progression," Journal of the National Cancer Institute, vol. 104, pp. 1825-1836, 2012.

[9] T. Gavressa, K. T. Kalogeras, G. A. Koliou, F. Zagouri, G. Lazaridis, and H. Gogas, "The prognostic value of the immunhistochemical expression of phosphorylated RB and p16 proteins in association with cyclin D1 and the p53 pathway in a large cohort of patients with breast cancer treated with taxane-based adjuvant chemotherapy," Anticancer Research, vol. 37, pp. 2947-2957, 2017.

[10] R. J. Duronio, P. H. O’Farrell, J. E. Xie, A. Brook, and N. Dyson, "The transcription factor E2F is required for S phase during Drosophila embryogenesis," Genes \& Development, vol. 9, no. 12, pp. 1445-1455, 1995.

[11] M. Derenzini, G. Donati, G. Mazzini et al., "Loss of retinoblastoma tumor suppressor protein makes human breast cancer cells more sensitive to antimetabolite exposure," Clinical Cancer Research, vol. 14, no. 7, pp. 2199-2209, 2008.

[12] E. Risi, A. Grilli, I. Migliaccio et al., "A gene expression signature of Retinoblastoma loss-of-function predicts resistance to neoadjuvant chemotherapy in ER-positive/ HER2-positive breast cancer patients," Breast Cancer Research and Treatment, vol. 170, no. 2, pp. 329-341, 2018.

[13] E. Risi, C. Biagioni, M. Benelli et al., "An RB-1 loss of function gene signature as a tool to predict response to neoadjuvant chemotherapy plus anti-HER2 agents: a substudy of the NeoALTTO trial (BIG 1-06)," Therapeutic advances in medical oncology, vol. 11, p. 1758835919891608, 2019.

[14] L. Malorni, S. Piazza, Y. Ciani et al., "A gene expression signature of retinoblastoma loss-of-function is a predictive biomarker of resistance to palbociclib in breast cancer cell lines and is prognostic in patients with ER positive early breast cancer," Oncotarget, vol. 7, no. 42, pp. 68012-68022, 2016.

[15] J. M. Patel, A. Goss, J. E. Garber et al., "Retinoblastoma protein expression and its predictors in triple-negative breast cancer," Npj Breast Cancer, vol. 6, no. 1, p. 19, 2020.

[16] D. Treré, E. Brighenti, G. Donati et al., "High prevalence of retinoblastoma protein loss in triple-negative breast cancers and its association with a good prognosis in patients treated with adjuvant chemotherapy," Annals of Oncology: Official Journal of the European Society for Medical Oncology, vol. 20, pp. 1818-1823, 2009.

[17] J. I. Herschkowitz, X. He, C. Fan, and C. M. Perou, "The functional loss of the retinoblastoma tumour suppressor is a common event in basal-like and luminal B breast carcinomas," Breast Cancer Research, vol. 10, no. 5, p. R75, 2008.

[18] F. Yang, Y. Wang, Q. Li et al., "Intratumor heterogeneity predicts metastasis of triple-negative breast cancer," Carcinogenesis, vol. 38, no. 9, pp. 900-909, 2017 Sep 1. 